\title{
Modification of the fatty acid composition in Arabidopsis and maize seeds using a stearoyl-acyl carrier protein desaturase-1 (ZmSAD1) gene
}

Hewei Du ${ }^{1,3^{*}}$, Min Huang ${ }^{1}$, Jieyun $\mathrm{Hu}^{2}$ and Jiansheng $\mathrm{Li}^{2}$

\begin{abstract}
Background: Stearoyl-acyl carrier protein desaturase (SAD) is a key enzyme that catalyses the conversion of stearoyl-acyl carrier protein (ACP) to oleoyl-ACP, a precursor for the biosynthesis of polyunsaturated fatty acids. ZmSAD1 (GenBank: KU949326) is a major QTL for stearic acid content in maize seeds. To investigate the biological function and the application potential of maize ZmSAD1 in oil biosynthesis, we isolated the full-length ZmSAD1 CDNA from maize B73 and overexpressed it in Arabidopsis and maize.

Results: Under seed-specific overexpression of ZmSAD1 in Arabidopsis, the stearic acid content and the ratio of saturated to unsaturated fatty acids in the seeds were significantly decreased relative to those in the control. Conversely, in transgenic ZmSAD1 RNAi Arabidopsis seeds, the contents of stearic acid and long-chain saturated acids and the ratio of saturated to unsaturated fatty acids were significantly increased; in addition, the oleic acid content was significantly decreased. More importantly, transgenic ZmSAD1 maize that expressed high levels of ZmSAD1 in its mature seeds showed reduced stearic acid content (1.57\%) and a lower saturated to unsaturated fatty acid ratio (20.40 \%) relative to those (1.64 \% and $20.61 \%$, respectively) of the control. Conversely, down-regulation of ZmSAD1 in maize resulted in increased levels of stearic acid (1.78\%), long-chain saturated acids $(0.85 \%)$ and the ratio of saturated to unsaturated fatty acids (21.54 \%) relative to those $(1.64 \%, 0.74 \%$, and $20.61 \%$, respectively) of the control, whereas the oleic acid (32.01\%) level was significantly decreased relative to that (32.68\%) of the control.
\end{abstract}

Conclusions: Our work demonstrates that the contents of stearic acid, oleic acid, and long-chain saturated acids, and the ratio of saturated to unsaturated fatty acids, are modified in maize seeds by seed-specific overexpression or down-regulation of ZmSAD1. Therefore, the ZmSAD1 gene is a useful tool for engineering the seed oil composition in maize and other crops.

Keywords: Maize (Zea mays L.), Arabidopsis, Stearoyl-acyl carrier protein desaturase, Oil engineering, Stearic acid, Oleic oil

\section{Background}

Triacylglycerides (TAG) is the main form of oil storage in many seeds, composed of fatty acids and glycerol, providing carbon and energy to support the establishment of seedlings after germination [1]. Seed oils are not

\footnotetext{
*Correspondence: duhewei666@163.com

${ }^{1}$ College of Life Science, Yangtze University, Jingzhou, Hubei 434025,

Peoples's Republic of China

${ }^{3}$ Hubei Collaborative Innovation Center for Grain Industry, Yangtze University, Jingzhou 434025, Peoples's Republic of China

Full list of author information is available at the end of the article
}

only a major food source for humans but can also serve as a desirable biofuel alternative to fossil oil worldwide $[2,3]$. Many crops, such as sesame, soybean, rapeseed, maize, sunflower, cotton, etc., produce vegetable oils in their seeds. However, the composition and content of fatty acids among these crops vary greatly. The quality and utilisation of seed oils is determined by their fatty acid composition. Saturated fatty acids, such as palmitic (C16:0) and stearic acids (C18:0), are stable and tolerant to heat and oxidation. Certain unsaturated fatty acids, 
such as oleic (C18:1), linoleic (C18:2), and linolenic (C18:3) acids, are beneficial to human health but susceptible to heat and oxidation [4]. The biosynthesis of fatty acids in plants has been extensively documented, which increases the feasibility of efficiently genetically engineering the composition of fatty acids in seeds.

Fatty acid biosynthesis in higher plants occurs in the chloroplasts of green tissues or in the plastids of nonphotosynthetic tissues [5]. In higher plants, the desaturation of long-chain fatty acids is accomplished in sequential steps and begins with the conversion of stearoyl-acyl carrier protein (ACP) to oleoyl-ACP, catalysed by stearoyl-ACP desaturase (SAD, EC 1.12.99.6) [6, 7]. Oleoyl-ACP is a precursor for the biosynthesis of polyunsaturated fatty acids [8]. The SAD enzyme introduces the first double bond into saturated fatty acids and is located on the plastid stroma. Therefore, SAD regulates the amount of unsaturated fatty acids and the ratio of saturated to unsaturated fatty acids in higher plants [9]. Several $S A D$ genes from a variety of plants have been isolated and characterised [7, 10-12]. Previous research has shown that the transference of a $S A D$ gene into Brassica napus and tobacco significantly increases the content of stearic and oleic acids [13, 14]. Maize seeds possess low stearic acid and high oleic acid contents; although oleic acid is beneficial to human health, they are sensitive to oxidation and unstable under high temperature conditions. $S A D$ may serve as a useful tool for engineering maize plants that produce a balanced content of stearic and oleic acids in their seeds.

The content and composition of oil in maize is a quantitative trait. Many QTLs associated with the content and composition of oil have been identified and mapped [15-17]. In our previous research, we combined two-dimensional gel electrophoresis (2-DGE) with mass spectrometry (MS) to compare the abundant soluble proteins of early developing embryos in maize. We identified 83 proteins that showed significantly different expression levels between a high-oil (By804) and a regular inbred line (B73), including the ZmSAD protein [18]. The expression level of ZmSAD (GenBank: DQ192663) was significantly higher in the By804 seeds than in the B73 seeds [18]. Maize contains two copies of the $Z m S A D$ gene, $Z m S A D 1$ and $Z m S A D 2$ that were isolated from By804 and B73 and mapped on bin3.05-3.06 and bin8.05-8.06, respectively [19]. The ZmSAD1 mRNA level was high in the B73 embryos but low in the By804 embryos; conversely, the ZmSAD2 mRNA level was high in the By804 embryos but low in the B73 embryos. $Z m S A D 1$ is one of the major QTLs that control stearic acid content [20]. ZmSAD2 is localized to the oilc8 QTL (phi119-umc1360) and accounts for $7.29 \%$ of the phenotypic variation in kernel oil concentration [17]. Because ZmSAD1 has a greater effect on oil content, we selected $Z m S A D 1$ to modify the composition of stearic and oleic acid in maize seeds.

For the genetic engineering of seed oil composition, ideal promoters should show high transcriptional activity and seed specificity. The Fatty Acid Elongation 1 (FAE1) promoter is seed specific [21]. For example, when the FAE1 promoter was used to control seed-specific overexpression of ZmSAD2, the composition of fatty acids in Arabidopsis seeds was desirably modified [22]. In this study, we tested the biological function and the potential application of ZmSAD1 in oil engineering. We transformed Arabidopsis and maize with ZmSAD1 using Agrobacterium-mediated transformation and particle bombardment, respectively, and succeeded in modifying the composition of fatty acids in maize seeds. Our results suggest that the $Z m S A D 1$ gene is a helpful tool for genetically engineering the composition of oil in maize or oil crops.

\section{Results \\ Endogenous $S A D$ expression levels are altered in transgenic Arabidopsis}

We obtained the full-length cDNA of ZmSAD1 from B73 using the 5'-RACE technique [19]. The ZmSAD1 overexpression (ZmSAD1), antisense ZmSAD1 (anti$Z m S A D 1)$, and ZmSAD1 RNA interference (ZmSAD1 RNAi) constructs were generated in the pBI121 vector (Additional file 1: Figure S1), and their expression was under control of the $F A E 1$ promoter, a seed-specific promoter [21]. The constructs were then used to transform Arabidopsis. To assess the expression levels of the exogenous ZmSAD1 gene in various Arabidopsis tissues, qRT-PCR experiments were performed. In the transgenic ZmSAD1 lines (\#1-1-5, 2-4-1, 3-5-2, 4-8-3), exogenous $Z m S A D 1$ exhibited significantly high expression levels in mature seeds and immature siliques than in roots, stems, leaves, and petals (Fig. 1). Without the presence of $Z m S A D 1$, the expression of $Z m S A D 1$ was undetectable in the WT and transgenic empty vector Arabidopsis lines (\#3-3-7) (Fig. 1). These results illustrate that the FAE1 promoter drives high expression levels of ZmSAD1 in immature siliques and mature seeds.

The Arabidopsis genome encodes seven homologous AtSAD genes: SSI2/FAB2 (GenBank: At2g43710), S-ACP$D E S 1$ (stearoyl-acyl carrier protein-desaturase) (GenBank: At5g16240), S-ACP-DES2 (GenBank: At3g02610), S-ACPDES3 (GenBank: At5g16230), S-ACP-DES4 (GenBank: At3g02620), S-ACP-DES5 (GenBank: At3g02630), and S-ACP-DES6 (GenBank: At1g43800) [23]. An alignment analysis of the deduced amino acid sequences of ZmSAD1 and the seven AtSAD genes was performed using ClustalX. The deduced amino sequences $(124 \sim 146$ aa, $170 \sim 196$ aa, $199 \sim 211$ aa, $215 \sim 241$ aa, $272 \sim 283$ aa) are highly conserved (Additional file 2: Figure S2). A phylogenetic relationship analysis shows that ZmSAD1 shares greater 


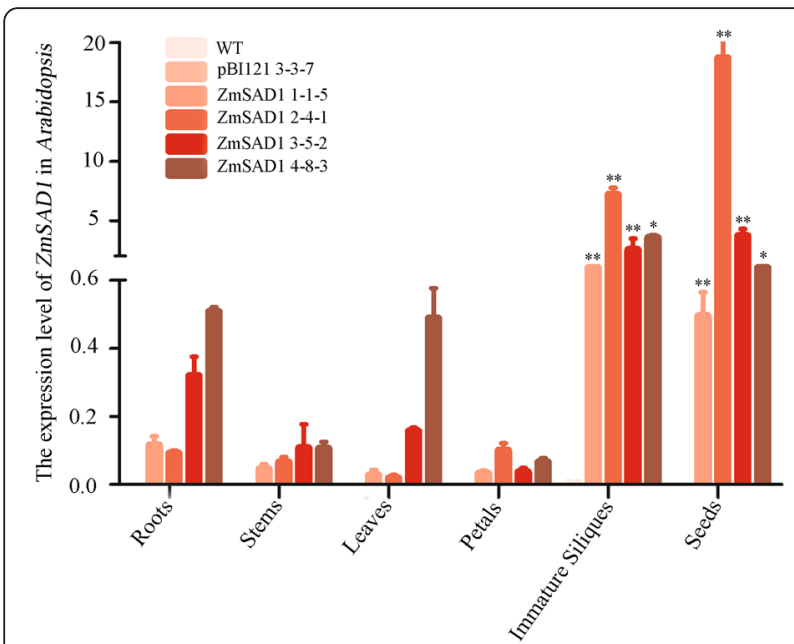

Fig. 1 Expression levels of exogenous $Z m S A D 1$ in various tissues of Arabidopsis. All of the experiments were performed with three biological replicates. In each transgenic line, approximately $0.2 \mathrm{~g}$ of roots, stems, and leaves were collected from 6 plants. Approximately $0.1 \mathrm{~g}$ petals and $0.2 \mathrm{~g}$ immature siliques were collected from 8 transgenic plants from each line. Approximately $0.1 \mathrm{~g}$ mature seeds collected from 18 transgenic plants were collected from each line. Total RNA samples were isolated from the above tissues. The expression level of ZmSAD1 was determined by qRT-PCR. The relative transcript levels were calculated using the Arabidopsis actin1 gene (GenBank: NM179953) as the internal reference. Each bar represents the mean \pm SD of three independent biological replicates. Student's $t$-test was performed. *indicates $p<0.05$; *indicates $p<0.01$

homology with SSI2/FAB2 compared with the other proteins (Fig. 2). To assess the expression level of the Arabidopsis endogenous AtSAD genes in the mature seeds, a qRT-PCR assay was performed. The results showed that SSI2/FAB2, S-ACP-DES2, and S-ACP-DES3 were expressed at lower levels than the other AtSAD genes in the mature seeds. In all cases, the expression levels of $S-A C P-D E S 2$, $S$-ACP-DES3 and $S$-ACP-DES4 remained unchanged upon introduction of a $Z m S A D 1$ construct. The expression of $S-A C P-D E S 1$ was significantly decreased in both the transgenic anti-ZmSAD1 and ZmSAD1 RNAi plants compared with the empty vector plant (Fig. 3). The expression of SSI2/FAB2 was slightly decreased in the transgenic anti-ZmSAD1 seeds compared with the control (transgenic empty vector), although the difference was not statistically significant. The expression levels of SSI2/FAB2, S-ACP-DES5 and S-ACP-DES6 were significantly decreased in the transgenic $Z m S A D 1$ RNAi seeds compared with that of control (Fig. 3a, f, g). These results confirm that ZmSAD1 RNAi and/or anti-ZmSAD1 suppressed the expression levels of SSI2/FAB2, S-ACP$D E S 1, S$-ACP-DES5, S-ACP-DES6, and SSI2/FAB2 in the transgenic Arabidopsis seeds.

\section{Fatty acid composition and content are modified in transgenic Arabidopsis seeds}

The compositions of fatty acids in the Arabidopsis seeds of transgenic lines were analysed by gas chromatography, together with the transgenic pBI121 empty vector line as the control. The contents of various fatty acids are summarized in Table 1 and Additional file 3: Table S1. With seed-specific ZmSAD1, the average content of stearic acid (2.88 \%) was significantly decreased compared with control (3.27 \%); conversely, with seed-specific silencing using ZmSAD1 RNAi, the average content of stearic acid (3.62 \%) was significantly increased compared with control (Table 1). However, the average contents of oleic acid in the anti-ZmSAD1 (13.94\%) and ZmSAD1 RNAi $(12.24 \%)$ lines were lower than in the control (15.61\%). The average content of long-chain saturated acids (2.54\%) in the ZmSAD1 plants was moderately reduced relative to the control (2.84\%), whereas it was significantly increased in the ZmSAD1 RNAi plants (3.25 \%) (Table 1). The ratio of saturated to unsaturated fatty acids was significantly reduced in the ZmSAD1 plants (17.28 \%); conversely, it was significantly increased in both the anti-ZmSAD1 (18.69\%) and ZmSAD1 RNAi plants (20.50 \%) (Table 1). Therefore, the seed-specific overexpression of the exogenous $Z m S A D 1$ gene significantly reduced the content of stearic acid and the ratio of saturated to unsaturated fatty acids. The seed-specific down-regulation of endogenous AtSAD genes in Arabidopsis significantly increased the content of stearic and long-chain saturated fatty acids and the ratio

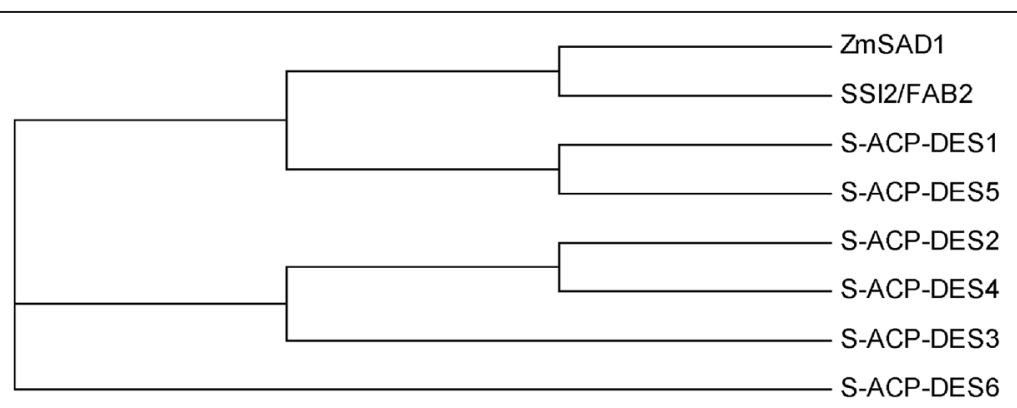

Fig. 2 Phylogenetic tree of ZmSAD1 and seven Arabidopsis AtSAD homologous genes. Multiple protein sequence alignment analyses were performed using Clustal $X$. The phylogenetic tree of the aligned protein sequences was constructed using MEGA 5.0 


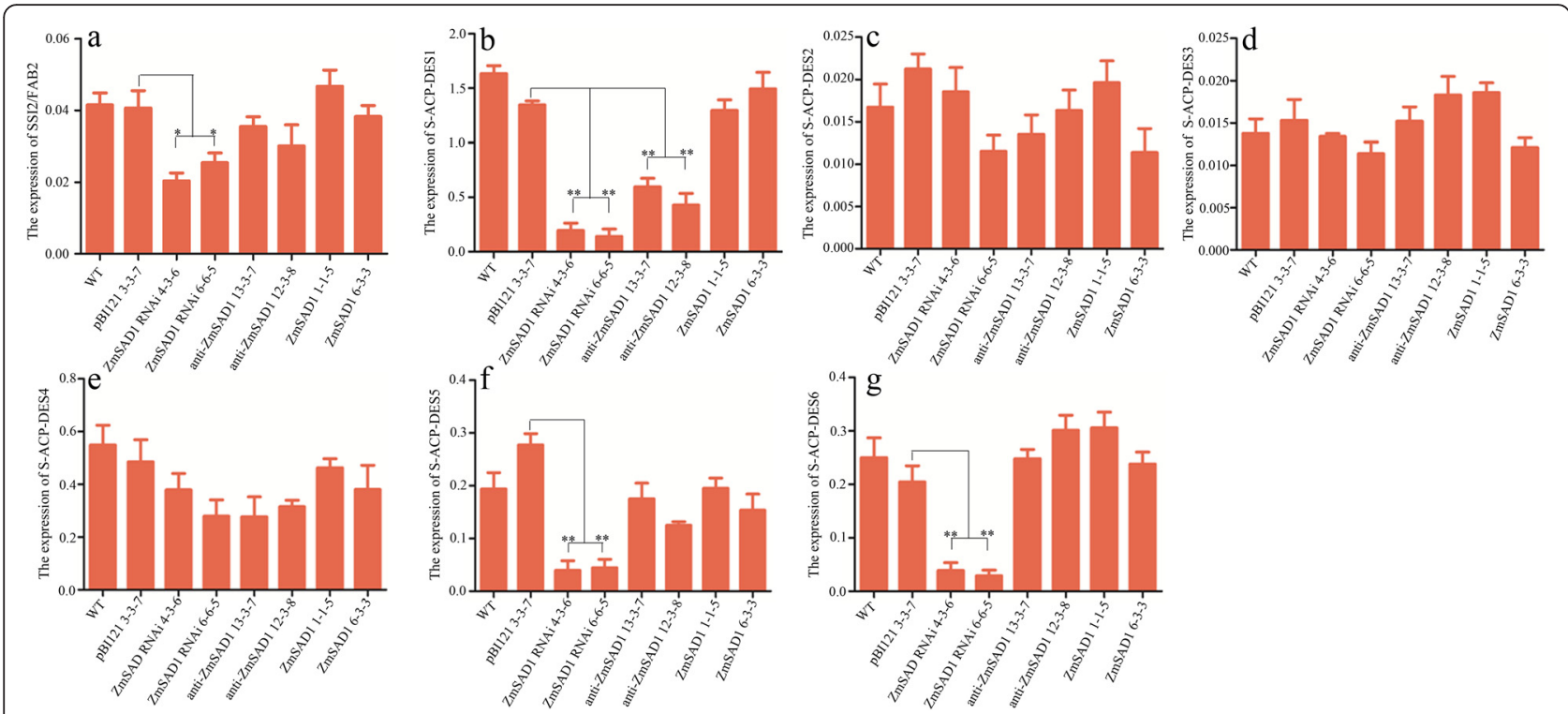

Fig. 3 Expression levels of the Arabidopsis endogenous AtSAD genes in mature seeds. a, SS12/FAB2. b, S-ACP-DES1. c, S-ACP-DES2. d, S-ACP-DES3. e, S-ACP-DES4. f, S-ACP-DES5. g, S-ACP-DES6. In each transgenic line, approximately $0.1 \mathrm{~g}$ mature seeds collected from 18 plants were used for total RNA isolation. The expression levels of the seven Arabidopsis endogenous AtSAD were determined by qRT-PCR. Expression levels were calculated using the Arabidopsis actin 1 gene as the internal reference. Each bar represents the mean \pm SD of three independent biological replicates. Student's t-test was performed. ${ }^{*}$ indicates $p<0.05 ;{ }^{* *}$ indicates $p<0.01$

Table 1 Composition of fatty acids in the Arabidopsis seeds harbouring the exogenous ZmSAD1, anti-ZmSAD1, or ZmSAD1 RNAi constructs

\begin{tabular}{|c|c|c|c|c|c|c|c|c|}
\hline \multirow[t]{2}{*}{ Lines } & \multicolumn{2}{|l|}{ Stearic acid } & \multicolumn{2}{|l|}{ Oleic acid } & \multicolumn{2}{|c|}{$\begin{array}{l}\text { Long-chain saturated acids } \\
\text { (\%) }\end{array}$} & \multicolumn{2}{|c|}{$\begin{array}{l}\text { Ratio of saturated to unsaturated fatty } \\
\text { acids (\%) }\end{array}$} \\
\hline & Content (\%) & Mean (\%) & Content (\%) & Mean (\%) & Content (\%) & Mean (\%) & Content (\%) & Mean (\%) \\
\hline pB|121 1-3-5 & $3.27 \pm 0.07$ & $3.27 \pm 0.19$ & $15.15 \pm 0.29$ & $15.61 \pm 0.87$ & $2.98 \pm 0.06$ & $2.84 \pm 0.21$ & $17.76 \pm 0.08$ & $17.71 \pm 0.04$ \\
\hline pB|121 3-3-7 & $3.47 \pm 0.10$ & & $16.62 \pm 0.05$ & & $2.57 \pm 0.07$ & & $17.72 \pm 0.16$ & \\
\hline pB|121 8-5-2 & $3.08 \pm 0.06$ & & $15.07 \pm 0.43$ & & $2.97 \pm 0.06$ & & $17.69 \pm 0.11$ & \\
\hline ZmSAD1 1-1-5 & $2.76 \pm 0.11$ & $2.88 \pm 0.21^{* *}$ & $14.94 \pm 0.39$ & $15.21 \pm 0.87$ & $2.66 \pm 0.11$ & $2.54 \pm 0.23$ & $16.77 \pm 0.15$ & $17.28 \pm 0.56^{*}$ \\
\hline ZmSAD1 2-4-1 & $3.02 \pm 0.07$ & & $14.04 \pm 0.11$ & & $2.51 \pm 0.21$ & & $16.82 \pm 0.16$ & \\
\hline ZmSAD1 3-5-2 & $3.12 \pm 0.30$ & & $15.64 \pm 0.23$ & & $2.40 \pm 0.04$ & & $18.02 \pm 0.28$ & \\
\hline ZmSAD1 4-8-3 & $2.71 \pm 0.06$ & & $14.84 \pm 0.37$ & & $2.63 \pm 0.03$ & & $17.04 \pm 0.27$ & \\
\hline ZmSAD1 6-3-3 & $2.67 \pm 0.08$ & & $15.15 \pm 0.33$ & & $2.58 \pm 0.13$ & & $17.08 \pm 0.59$ & \\
\hline ZmSAD1 7-2-4 & $3.01 \pm 0.08$ & & $16.62 \pm 0.46$ & & $2.48 \pm 0.16$ & & $17.97 \pm 0.88$ & \\
\hline Anti-ZmSAD1 2-8-5 & $3.26 \pm 0.12$ & $3.31 \pm 0.15$ & $15.51 \pm 0.21$ & $13.94 \pm 1.66^{*}$ & $2.46 \pm 0.14$ & $2.82 \pm 0.40$ & $18.39 \pm 0.03$ & $18.69 \pm 0.58^{* *}$ \\
\hline Anti-ZmSAD1 16-5-1 & $3.45 \pm 0.07$ & & $15.96 \pm 0.23$ & & $2.82 \pm 0.08$ & & $18.01 \pm 0.83$ & \\
\hline Anti-ZmSAD1 13-3-7 & $3.49 \pm 0.06$ & & $13.11 \pm 0.07$ & & $2.39 \pm 0.02$ & & $19.47 \pm 0.09$ & \\
\hline Anti-ZmSAD1 18-6-2 & $3.15 \pm 0.09$ & & $12.73 \pm 0.28$ & & $3.19 \pm 0.06$ & & $19.09 \pm 0.61$ & \\
\hline Anti-ZmSAD1 30-2-2 & $3.20 \pm 0.01$ & & $12.41 \pm 0.26$ & & $3.26 \pm 0.04$ & & $18.51 \pm 0.32$ & \\
\hline ZmSAD1 RNAi 2-2-7 & $3.21 \pm 0.08$ & $3.62 \pm 0.37^{*}$ & $11.77 \pm 0.25$ & $12.24 \pm 0.53^{* *}$ & $3.51 \pm 0.08$ & $3.25 \pm 0.20^{* *}$ & $19.60 \pm 0.21$ & $20.50 \pm 2.17^{* *}$ \\
\hline ZmSAD1 RNAi 4-3-6 & $4.3 \pm 0.09$ & & $11.58 \pm 0.16$ & & $3.04 \pm 0.11$ & & $23.95 \pm 0.19$ & \\
\hline ZmSAD1 RNAi 6-6-5 & $3.7 \pm 0.05$ & & $12.56 \pm 0.05$ & & $3.37 \pm 0.08$ & & $21.14 \pm 0.18$ & \\
\hline ZmSAD1 RNAi 7-4-4 & $3.20 \pm 0.09$ & & $12.80 \pm 0.24$ & & $3.19 \pm 0.03$ & & $18.33 \pm 0.42$ & \\
\hline ZmSAD1 RNAi 12-1-5 & $3.69 \pm 0.08$ & & $12.50 \pm 0.08$ & & $3.17 \pm 0.12$ & & $19.49 \pm 0.78$ & \\
\hline
\end{tabular}


of saturated to unsaturated fatty acids, whereas it significantly decreased the oleic acid content.

\section{Introduction of the ZmSAD1 gene into maize}

Our results confirmed that the composition of fatty acids in the Arabidopsis seeds was modified by the introduction of an exogenous $Z m S A D 1$ gene and suppressed by ZmSAD1 RNAi. Because maize oils are one of the major polyunsaturated vegetable oils for human consumption and modification of maize oil composition carries tremendous implications in practical application, we expanded our experiments to maize to modify the composition of fatty acids in maize seeds. We individually transformed the $Z m S A D 1$ and ZmSAD1 RNAi constructs under control of the $F A E 1$ promoter into maize by particle bombardment. Maize calli resistant to bialaphos selection were obtained after particle bombardment (Additional file 4: Figure S3a), and plantlets were regenerated from these calli. The plantlets with well-developed roots were transferred to a growth chamber (Additional file 4: Figure S3b). PCR and Southern blot assays were conducted; the results confirmed that the $Z m S A D 1$ gene was integrated into the maize genome (Additional file 4: Figure S3c, d). Subsequently, positive ZmSAD1 and $Z m S A D 1$ RNAi $\mathrm{T}_{0}$ plants were crossed with inbred line A188. The ZmSAD1 and ZmSAD1 RNAi constructs were established in the A188 background (sharing approximate 99.4 \% similarity) after five back crosses with the recurrent parent A188 followed by one self-pollination. We obtained four A188(ZmSAD1) and three A188(ZmSAD1 RNAi) lines. The ZmSAD1 expression levels in the maize mature embryos were determined using qRT-PCR. The total $Z m S A D 1$ expression levels (including endogenous $Z m S A D 1$ and exogenous $Z m S A D 1)$ in the A188(ZmSAD1) mature embryos are higher compared with that of the control. In contrast, the ZmSAD1 expression levels in the A188(ZmSAD1 RNAi) mature seeds are lower than that in the control, indicating that the ZmSAD1 RNAi construct effectively suppresses expression of the endogenous $Z m S A D 1$ gene in maize (Fig. 4).

\section{Overexpression of ZmSAD1 changes the fatty acid composition in maize seeds}

The composition of fatty acids in maize seeds was also analysed using gas chromatography. The contents of various fatty acids are shown in Table 2 and Additional file 5: Table S2. In A188 seeds, the percentage of oleic acid in total fatty acids is $32.68 \%$ (Table 2), which is higher than the percentage of other fatty acids. In the A188(ZmSAD1) mature seeds (\#1-5, 2-3, 3-1, 4-5), the average content of stearic acid $(1.57 \%)$ and the ratio of saturated to unsaturated acids (20.40\%) are significantly decreased compared with those of the A188 control (1.64 \% and $20.61 \%$, respectively) (Table 2), indicating

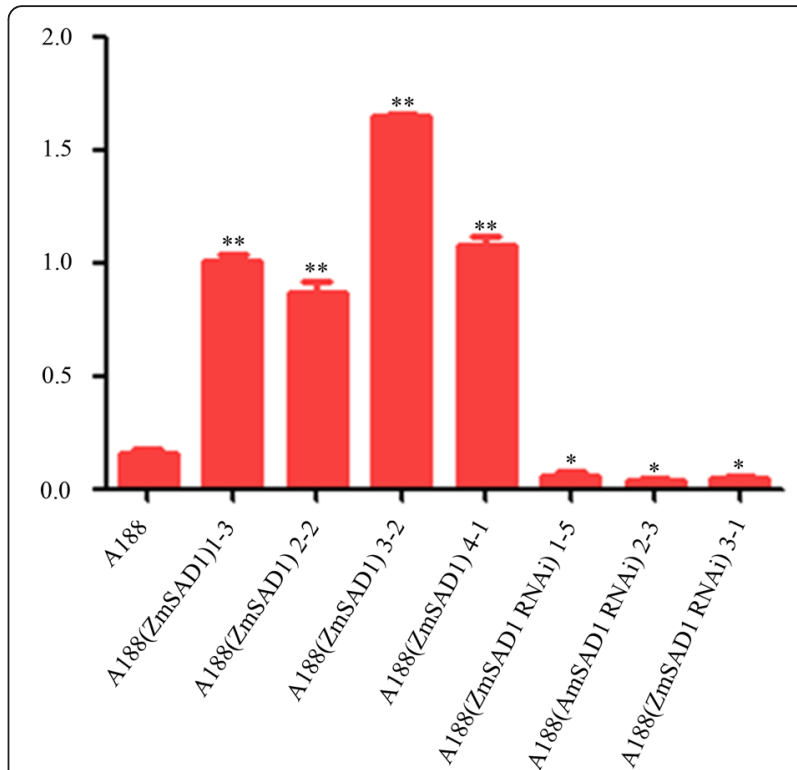

Fig. 4 Expression levels of ZmSAD1 in the mature maize embryos. Ten maize embryos were excised from the mature seeds of A188, A188(ZmSAD1), and A188(ZmSAD1 RNAi). Total RNA samples were isolated from mature embryos. The expression level of ZmSAD1 was determined for each sample by qRT-PCR. Three biological replicates were performed. The expression levels were calculated using the maize actin 1 gene (MaizeGDB: GRMZM2G126010) as the internal reference. Each bar represents the mean \pm SD of three independent biological replicates. Student's $t$-test was performed. ${ }^{*}$ indicates $p<0.05 ;{ }^{* *}$ indicates $p<0.01$

that seed-specific $Z m S A D 1$ results in a decrease in the content of stearic and other saturated acids. On the contrary, seed-specific down-regulation of ZmSAD1 by RNAi increases the average content of stearic acid (1.78\%) and decreases the average content of oleic acid (32.01\%) compared with those of the control $(1.64 \%$ and $32.68 \%$, respectively) (Table 2). The average content of long-chain saturated fatty acids and the ratio of saturated to unsaturated fatty acids are both significantly increased in the A188(ZmSAD1 RNAi) mature seeds (\#1-2, 2-3, 3-3). Conversely, the contents of oleic acid and long-chain saturated acids in the A188(ZmSAD1) mature seeds are not significantly changed compared with those of the control.

\section{Discussion}

Reverse and forward genetics are widely used approaches to identify genes of interest. Our group has previously used a proteomics approach by conducting 2-dimensional gel electrophoresis to screen for differentially expressed proteins in the seeds of low and high-oil maize lines. Among the proteins identified in this proteomics study, the ZmSAD protein is expressed at high levels in By804 (a high-oil inbred line) and at low levels in B73 (a regular inbred line) [18]. Therefore, we selected $Z m S A D$ as 
Table 2 Composition of fatty acids in the maize seeds harbouring ZmSAD1 or ZmSAD1 RNAi constructs

\begin{tabular}{|c|c|c|c|c|c|c|c|c|}
\hline \multirow[t]{2}{*}{ Lines } & \multicolumn{2}{|l|}{ Stearic acid } & \multicolumn{2}{|l|}{ Oleic acid } & \multicolumn{2}{|c|}{$\begin{array}{l}\text { Long-chain saturated acids } \\
\text { (\%) }\end{array}$} & \multicolumn{2}{|c|}{$\begin{array}{l}\text { Ratio of saturated to unsaturated } \\
\text { fatty acids (\%) }\end{array}$} \\
\hline & Content (\%) & Mean (\%) & Content (\%) & Mean (\%) & Content (\%) & Mean (\%) & Content (\%) & Mean (\%) \\
\hline A188 & $1.64 \pm 0.03$ & $1.64 \pm 0.03$ & $32.68 \pm 0.09$ & $32.68 \pm 0.09$ & $0.74 \pm 0.01$ & $0.74 \pm 0.01$ & $20.61 \pm 0.38$ & $20.61 \pm 0.38$ \\
\hline A188(ZmSAD1) 1-5 & $1.57 \pm 0.02$ & $1.57 \pm 0.04^{*}$ & $32.39 \pm 1.02$ & $32.85 \pm 0.47$ & $0.69 \pm 0.04$ & $0.71 \pm 0.02$ & $20.48 \pm 0.97$ & $20.40 \pm 0.19^{*}$ \\
\hline A188(ZmSAD1) 2-3 & $1.52 \pm 0.06$ & & $32.51 \pm 0.53$ & & $0.73 \pm 0.03$ & & $20.63 \pm 0.41$ & \\
\hline A188(ZmSAD1) 3-1 & $1.58 \pm 0.02$ & & $33.11 \pm 0.82$ & & $0.71 \pm 0.02$ & & $20.24 \pm 0.14$ & \\
\hline A188(ZmSAD1) 4-5 & $1.61 \pm 0.05$ & & $33.37 \pm 1.07$ & & $0.71 \pm 0.01$ & & $20.25 \pm 0.26$ & \\
\hline A188(ZmSAD1 RNAi) 1-2 & $1.73 \pm 0.03$ & $1.78 \pm 0.05^{*}$ & $32.03 \pm 0.61$ & $32.01 \pm 0.17^{* *}$ & $0.86 \pm 0.05$ & $0.85 \pm 0.04^{*}$ & $21.29 \pm 0.37$ & $21.54 \pm 0.41^{* *}$ \\
\hline A188(ZmSAD1 RNAi) 2-3 & $1.79 \pm 0.01$ & & $32.17 \pm 0.27$ & & $0.88 \pm 0.03$ & & $21.32 \pm 0.26$ & \\
\hline A188(ZmSAD1 RNAi) 3-3 & $1.82 \pm 0.02$ & & $31.83 \pm 0.72$ & & $0.81 \pm 0.06$ & & $22.01 \pm 0.11$ & \\
\hline
\end{tabular}

Student's $t$-test was performed. *indicates $p<0.05 ;{ }^{* *}$ indicates $p<0.01$

candidate genes to determine their association with the amount and composition of fatty acids. Subsequently, the cDNA sequence of the ZmSAD (GenBank: DQ192663) gene was also annotated by an in silico approach [18]; the initial in silico gene prediction was further improved. Two copies of the ZmSAD gene, ZmSAD1 and ZmSAD2, were isolated by RACE from B73 and By804, respectively, and localized on chromosomes 3 and 8, respectively. The cDNA and deduced amino acid sequences of ZmSAD1 and $Z m S A D 2$ are highly homologous [19]. In our previous and current studies, the full-length cDNAs of ZmSAD1 and $Z m S A D 2$, both under control of the FAE1 promoter, were used to transform Arabidopsis. Overexpression of either $Z m S A D 1$ or $Z m S A D 2$ leads to a decrease in stearic acid content in Arabidopsis seeds (Table 1) [22]. However, whether $Z m S A D 1$ or $Z m S A D 2$ is the major gene regulating stearic acid content remains unclear. Later, through mapping, ZmSAD1 was confirmed to be the major QTL for the content of stearic acid [20]. Therefore, reverse genetics combined with forward genetics proved to be an effective solution in this case.

Stearic acid and oleic acid are regarded as two desirable dietary components of seed oil. Stearic acid has a higher melting point and is more resistant to oxidation compared with unsaturated acids, rendering it suitable for cooking, roasting, and frying [24]. In addition, stearic acid has a neutral effect on total and low-density lipoprotein (LDL) cholesterol levels in the blood [25]. As a monounsaturated fatty acid, oleic acid is beneficial to human health [25]. In this study, we examined the feasibility of modifying the fatty acid composition of maize by elevating or suppressing the expression level of the ZmSAD1 gene. A188(ZmSAD1 RNAi) seeds have a higher content of stearic acid and long-chain saturated acids and a lower content of oleic acid, whereas A188(ZmSAD1) seeds contain lower levels of stearic acid. However, the modified traits of A188(ZmSAD1), including contents of stearic acid, long-chain saturated acids, and oleic acid, are not the exact opposite to those of A188(ZmSAD1 RNAi). The content of long-chain saturated acids was decreased in A188(ZmSAD1), although the change was not statistically significant.

The contents of stearic acid and oleic acid are approximately $1.64 \%$ and $32.68 \%$, respectively, in A188 maize; thus oleic acid is about 20 times higher than stearic acid in A188. With the seed-specific overexpression of ZmSAD1, the average content of stearic acid in A188(ZmSAD1) was significantly decreased (by $0.07 \%$ ) to $1.57 \%$. The magnitude of this decrease in stearic acid content is small compared with the oleic acid content in A188(ZmSAD1). It also resulted in an insignificant increase in the oleic acid content, insufficient to significantly improve the oleic acid content. Similar results were also found in the Arabidopsis transgenic ZmSAD1 plants. Therefore, we hypothesize that introduction of multiple genes to engineer the fatty acid synthesis pathway is required in order to effectively modify the composition of multiple fatty acids.

The SAD enzyme catalyses the desaturation of stearoyl-ACP to form oleoyl-ACP. ZmSAD1 is highly homologous to ZmSAD2 [19], which indicates a functional similarity between the two genes encoding the proteins. However, ZmSAD1 is a major QTL for stearic acid, whereas ZmSAD2 is a QTL for oil content [19]. The difference in the biological functions of ZmSAD1 and ZmSAD2 may be associated with their expression levels and their genetic backgrounds. For example, the maize inbred line A188 contains endogenous ZmSAD1. With the seed-specific overexpression of ZmSAD1, the content of stearic acid and the ratio of saturated to unsaturated fatty acids were successfully modified in A188(ZmSAD1) mature seeds (Table 2), suggesting that the elevated level of $Z m S A D 1$ is an effective solution for decreasing the stearic acid content in the A188 background. The expression levels of ZmSAD1 allelic genes vary significantly in different maize genetic backgrounds. Thus, inbred lines with high ZmSAD1 expression levels 
may be the best candidates for modifying the fatty acid composition by traditional breeding.

Antisense and RNAi approaches have been used to suppress the expression of endogenous genes and to modify fatty acid compositions [26, 27]. However, the degree of gene silencing under RNAi-mediated suppression is much higher than that under antisense-mediated suppression, particularly in heterozygotes [28]. In the present study, we individually introduced into Arabidopsis the anti-ZmSAD1 and ZmSAD1 RNAi constructs under control of the same $F A E 1$ promoter. However, the fatty acid modification in the Arabidopsis transgenic anti-ZmSAD1 seeds was not consistent with that in the ZmSAD1 RNAi seeds. We hypothesize that this discrepancy may be due to the degree of silencing of endogenous AtSAD between anti-ZmSAD1 and ZmSAD1 RNAi seeds. Among the 7 Arabidopsis $S A D$ genes, SI2/FAB2, S-ACP-DES1, $S$-ACP-DES5, and $S$-ACP-DES6 expression levels were significantly decreased in the transgenic ZmSAD1 RNAi seeds, whereas only the $S$-ACP-DES1 level was decreased in the transgenic anti-ZmSAD1 seeds, which confirmed that the ZmSAD1 RNAi construct is more effective than the anti-ZmSAD1 construct in silencing AtSAD genes.

All seven Arabidopsis SAD enzymes utilize C18:0-ACP as the preferred substrate [23], which indicates that these proteins can catalyse the desaturation of C18:0-ACP to C18:1-ACP. In Arabidopsis transgenic ZmSAD1 RNAi seeds, the SSI2/FAB2, S-ACP-DES1 and S-ACP-DES5 levels were significantly decreased, indicating that the C18:0-ACP to C18:1-ACP conversion step in fatty acid synthesis was suppressed. On the contrary, in antiZmSAD1 Arabidopsis seeds, although the S-ACP-DES1 level was significantly decreased, the SSI2/FAB2 and $S$ $A C P$-DES5 levels remained unchanged. The unchanged $S S I 2 / F A B 2$ and $S-A C P-D E S 5$ levels probably compensated for the decrease of $S-A C P-D E S 1$ in anti-ZmSAD1 seeds, thereby resulting in no changes in the content of stearic and long-chain saturated fatty acids.

\section{Conclusions}

In this study, ZmSAD1 and ZmSAD1 RNAi constructs were transformed into maize; the composition of fatty acids was analysed in mature maize seeds using gas chromatography. With seed-specific overexpression of ZmSAD1, the content of stearic acid and the ratio of saturated to unsaturated fatty acids are significantly decreased. Down-regulation of ZmSAD1 results in increased levels of stearic acid and long-chain saturated acids and increased ratio of saturated to unsaturated fatty acids, whereas the content of oleic acid is significantly decreased. We have demonstrated that the $Z m S A D 1$ gene is an effective tool for modifying the oil composition of maize or oil crops.

\section{Methods}

Plant growth

Arabidopsis seeds were treated with $70 \%$ ethanol for one minute, soaked in $1 \%$ sodium hypochlorite for $15 \mathrm{~min}$, and rinsed with sterile water 4-5 times. The sterilised seeds were transferred to MS medium and grown in a chamber at $19-21{ }^{\circ} \mathrm{C}$ for 10 days. Ten-day old seedlings were planted at a density of 2 plants per $25 \mathrm{~cm}^{2}$ in moistened potting soil covered with a nylon window screen. The seedlings were grown in a growth chamber at $19-21{ }^{\circ} \mathrm{C}$ with a $16 \mathrm{~h} / 8 \mathrm{~h}$ light-dark cycle.

Maize plantlets derived from the resistant calli were grown in MS medium for 15 days. The plantlets with well-developed roots were transferred into pots with moistened soil. False-positive transgenic seedlings were excluded by performing a PCR screen. Positive transgenic plants were planted in a greenhouse at $23-25{ }^{\circ} \mathrm{C}$ with a $16 \mathrm{~h} / 8 \mathrm{~h}$ light-dark cycle.

\section{Binary construct and Agrobacterium strain}

The FAE1 (GenBank: AF355982.1) promoter was isolated from Arabidopsis genomic DNA by PCR amplification $[19,20]$, and it was substituted for the CaMV35S promoter in the pBI121 vector. Full-length ZmSAD1 cDNA derived from B73, the antisense ZmSAD1 construct (full-length ZmSAD1 cDNA), and the ZmSAD1 RNAi construct (part sequence of ZmSAD1 cDNA) (Additional file 4: Figure S3a, b, c) were cloned into the pBI121 binary vector and the pCAMBIA3301 vector (Additional file 4: Figure S3d, e), which were both under control of the FAE1 promoter. The pBI121 vector contains a Nos promoter-NPT II gene cassette and the pCAMBIA3301 vector contains a CaMV 35S promoter-bar gene cassette for the selection of transformants. Partial maps of the resulting constructs as well as the loop sequence and part of $Z m S A D 1$ in the RNAi construct are shown in Additional file 1: Figure S1. The pBI121ZmSAD1, pBI121-anti-ZmSAD1, pBI121-ZmSAD1 RNAi, and empty pBI121 vector were mobilized individually into Agrobacterium GV3101 by a direct DNA transfer method [29], and their integrity in the Agrobacterium cells was confirmed by restriction enzyme analysis after extraction of the plasmid from GV3101.

\section{Arabidopsis and maize transformation}

Arabidopsis transformation was conducted using the floral dip method according to Clough and Bent [30]. Mature seeds were harvested, dried down, and stored until selection. The seeds were treated with $70 \%$ ethanol for $1 \mathrm{~min}$ and $1 \%$ sodium hypochlorite for $15 \mathrm{~min}$ and then rinsed five times with sterile water. The sterilized seeds were planted on MS medium with kanamycin $\left(75 \mu \mathrm{g} \mathrm{ml}^{-1}\right)$ and grown in a chamber at $21{ }^{\circ} \mathrm{C}$ under a $16 \mathrm{~h}$ light (50-100 $\mu$ Einsteins $\left.\mathrm{m}^{-2} \mathrm{~s}^{-1}\right)$ cycle for 10 days. 
Kanamycin-resistant seedlings with green leaves and well-established roots were selected from the plates and planted into moistened potting soil.

Maize calli derived from immature Hi-II zygotic embryos were used as the target tissues for bombardment. The bombardment and subsequent selection of bialaphosresistant calli were performed according to Frame et al. [31]. Briefly, the calli were transferred to osmotic medium and then bombarded two times. A total of 40 petri dishes of calli were chosen for bombardment with the ZmSAD1 or ZmSAD1 RNAi construct. The bombarded calli were incubated at $28{ }^{\circ} \mathrm{C}$ in the dark. After selection and regeneration, the plantlets grew out from the bar-resistant calli. Genomic DNA was isolated from the leaves of the putative transgenic maize plantlets, and PCR and Southern blot assays were performed to confirm the presence of transgenes. Twenty micrograms of each DNA sample was digested with EcoRI at $37{ }^{\circ} \mathrm{C}$ overnight. The digestion products were fractionated on a $0.8 \%$ agarose gel and then transferred to a nylon membrane. The FAE1 promoter probe was labelled with DIG using the PCR method. A Southern blot analysis was conducted according to the protocol of the Roche Southern Blot Kit (Roche Applied Science, Mannheim, Germany). Welldeveloped positive $\mathrm{T}_{0}$ transgenic ZmSAD1 and ZmSAD1 RNAi plants were used as the donor to cross with maize inbred line A188, which was used as the recurrent parent. After five backcrosses and one self-pollination coupled with molecular marker-assisted selection for the transgene, the ZmSAD1 and ZmSAD1 RNAi constructs were introduced into the A188 genetic background.

\section{ZmSAD1 expression}

All of the experiments were performed with three biological replicates. Ninety-six well-developed seedlings from each homozygous transgenic $\mathrm{T}_{3}$ line of Arabidopsis were planted in a growth chamber. After four weeks, approximately $0.2 \mathrm{~g}$ of the roots, leaves, and stems were collected from 6 plants. At the flowering stage, approximately $0.1 \mathrm{~g}$ of the petals and $0.2 \mathrm{~g}$ of the immature siliques were collected from 8 plants. The seeds from each homozygous line were collected from 18 plants and pooled. Approximately $0.1 \mathrm{~g}$ of the mature seeds were used for further RNA isolation. In maize, 10 mature embryos from each line were excised from the transgenic or A188 seeds and used for RNA isolation.

Total RNA was isolated from various Arabidopsis tissues or maize mature embryos using TRIzol reagent (Invitrogen, Carlsbad, CA, USA). First-strand cDNA was synthesised using SuperScript-II reverse transcriptase according to the manufacturer's instructions (Invitrogen). The actin1 genes of Arabidopsis and maize (GenBank: NM179953 and MaizeGDB: GRMZM2G126010) were used as the endogenous controls to normalize the expression data (Additional file 6: Table S3). Real-time PCR was conducted using the SYBR Real-time PCR kit (Takara Japan) with IQTM SYBR ${ }^{\circ}$ Green Supermixture according to the manufacturer's instructions (Bio-Rad USA). The reaction condition was as follows: $94{ }^{\circ} \mathrm{C}$ for $1 \mathrm{~min}$, followed by $40 \mathrm{cy}$ cles of $95{ }^{\circ} \mathrm{C}$ for $10 \mathrm{~s}, 55^{\circ} \mathrm{C}$ for $10 \mathrm{~s}$, and $72{ }^{\circ} \mathrm{C}$ for $15 \mathrm{~s}$.

\section{Determination of fatty acid composition and content} Arabidopsis seeds were harvested from homozygous $\mathrm{T}_{3}$ transgenic plants. Mature seeds from each transgenic line were collected from 18 plants, pooled, and subjected to a fatty acid analysis. Ten maize seeds from each line were subjected to a lipid analysis. Seeds of the transgenic pBI121 empty vector in Arabidopsis and maize inbred line A188 were used as the controls. Approximately $0.1 \mathrm{~g}$ of ground seeds of Arabidopsis and $0.2 \mathrm{~g}$ of ground seeds of maize were used for each assay. All of the experiments were performed with three biological repeats. The total lipid was extracted from the ground seeds and content analysed as described by Sukhija and Palmquist [32]; the fatty acid composition was analysed using gas chromatography (Hewlett-Packard, Palo Alto, CA, USA). In the present study, the long-chain saturated acids include C20:0, C22:0, and C24:0; the saturated fatty acids include C14:0, C16:0, C18:0, C20:0, C22:0, and C24:0; the unsaturated fatty acids include C16:1, C18:1, C18:2, and $\mathrm{C} 18: 3$. Fatty acids that are present at low levels, including C10:0, C12:0, were not considered in this study. The content of each fatty acid was expressed as the percentage of total fatty acids.

\section{Sequence alignment and phylogenetic tree construction} Alignment analyses of ZmSAD1 and the seven AtSAD protein sequences were performed using ClustalX [33]. Phylogenetic trees of the aligned ZmSAD1 and AtSAD protein sequences were constructed using MEGA version 5.0 [34] via the neighbour-joining (NJ) method with the following parameters: Poisson correction, pairwise deletion, and bootstrapping (1000 replicates; random seeds).

\section{Additional files}

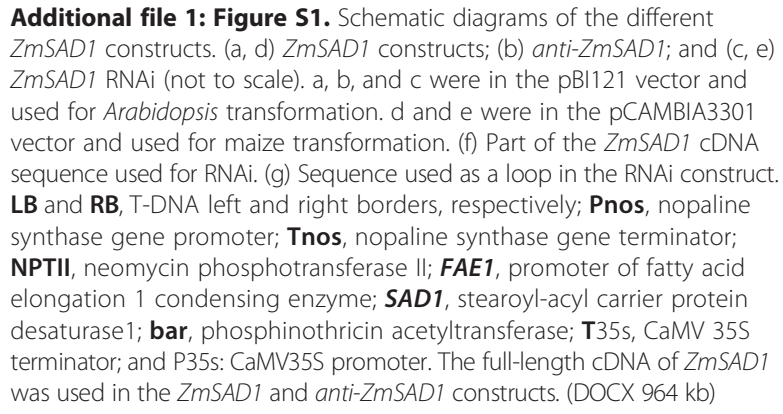


Additional file 2: Figure S2. Protein sequence alignment of ZmSAD1 and seven Arabidopsis AtSAD genes. Multiple protein sequence alignment analyses were performed using Clustal X. GenBank: At2g43710 (SS/2/FAB2), At5g16240 (S-ACP-DES1), At3g02610 (S-ACP-DES2), At5g16230 (S-ACP-DES3), At3g02620 (S-ACP-DES4), At3g02630 (S-ACP-DES5), At1g43800 (S-ACP-DES6), and ZmSAD1 (GenBank: KU949326). Positions are indicated relative to the ZmSAD1 protein sequence. (TIF $2280 \mathrm{~kb}$ )

Additional file 3: Table S1. Composition of fatty acids in the transgenic ZmSAD1 Arabidopsis mature seeds (DOCX $17 \mathrm{~kb}$ )

Additional file 4: Figure S3. Generation and confirmation of transgenic ZmSAD1 and ZmSAD1 RNAi maize plants. a, Resistant calli (indicated by arrows). b, Regenerated plantlets grown in a growth chamber. c, Detection of the transgene. Lane 1, DL2000 DNA marker. Lane 2, positive control (pCAMBIA3301-FAE1-ZmSAD1 plasmid). Lane 3, negative control (maize inbred line A188). Lane 4-13, transgenic ZmSAD1 plants. Lane 14, null. Lane 15-24, transgenic ZmSAD1 RNAi plants. Genomic DNA was isolated from the leaves of $T_{0}$ transgenic plants and used in the PCR and Southern blot analyses. d, Southern blot analysis. Lane 1, גDNA/Hindlll marker. Lane 2, pCAMBIA3301-FAE1-ZmSAD1 digested with EcoRI. Lane 3, non-transgenic plant (maize inbred line A188). Lane 4-5, positive transgenic plants. The FAE1 fragment was labelled with DIG via PCR amplification and used as probe in the Southern blot analysis. (TIF $5092 \mathrm{~kb}$ )

Additional file 5: Table S2. Composition of fatty acids in the transgenic ZmSAD1 maize seeds. (DOCX $15 \mathrm{~kb}$ )

Additional file 6: Table S3. Primers used in this study. (DOCX $14 \mathrm{~kb}$ )

\section{Abbreviations}

2-DGE, two-dimensional gel electrophoresis; ACP, acyl carrier protein; CaMV35S, CaMV35S promoter; CDS, coding sequence; DIG, digoxin; FAE1, Fatty Acid Elongation 1; MS, mass spectrometry; qRT-PCR, quantitative real time RT-PCR; RACE, rapid-amplification of CDNA ends; SAD and S-ACP-DES, Stearoyl-acyl carrier protein desaturase; TAG, triacylglycerides

\section{Acknowledgements}

We thank Dr. Zhizhong Chen (China Agricultural University) for providing the GV3101 strain; Dr. Xiangbo Xu (China Agricultural University) for providing the pCAMBIA3301 vector; and Dr. Feng Xu (Yangtze University) for providing the pBI121 vector. We are grateful to Dr. Mawsheng Chern (University of California at Davis) for his critical reading and editing of the manuscript.

\section{Funding}

This work was supported by the National Natural Science Foundation

(31271741) and the Hubei Province Natural Science Foundation

(2011CDB006 and 2012FFA051).

\section{Availability of data and materials}

The data sets supporting the results of this article are included within the article and its additional files.

\section{Authors' contributions}

LS designed the study. HWD performed the transformation of Arabidopsis and maize and analysed the fatty acid composition of Arabidopsis and maize seeds. HJY isolated the full-length CDNA of SADI from B73. HM backcrossed the maize SAD1 and SAD1 RNAi To plants using A188. HWD wrote the paper. All authors read and approved the final manuscript.

\section{Competing interests}

The authors declare that they have no competing interests.

\section{Consent for publication}

Not applicable.

\section{Ethics approval and consent to participate}

Not applicable.

\section{Author details}

'College of Life Science, Yangtze University, Jingzhou, Hubei 434025, Peoples's Republic of China. ${ }^{2}$ China Agricultural University, National Maize Improvement Center of China, Beijing 100193, Peoples's Republic of China.
${ }^{3}$ Hubei Collaborative Innovation Center for Grain Industry, Yangtze University, Jingzhou 434025, Peoples's Republic of China.

Received: 21 April 2016 Accepted: 7 June 2016

Published online: 14 June 2016

\section{References}

1. Bewley JD, Black M. Seed: physiology of development and germination. 2nd ed. New York: Plenum; 1994.

2. Troncoso-Ponce MA, Kilaru A, Cao X, Durrett TP, Fan JL, Jensen JK, Thrower NA, Pauly M, Wilkerson C, Ohlrogge JB. Comparative deep transcriptional profiling of four developing oilseeds. Plant J. 2011;68(6):1014-27.

3. Yang P, Shu C, Chen L, Xu JS, Wu JS, Liu KD. Identification of a major QTL for silique length and seed weight in oilseed rape (Brassica napus $\mathrm{L}$.). Theor Appl Genet. 2012;125(2):285-96.

4. Hu FB, Stampfer MJ, Manson JE, Rimm E, Colditz GA, Rosner BA, Hennekens $\mathrm{CH}$, Willett WC. Dietary fat intake and the risk of coronary heart disease in women. N Engl J Med. 1997;337:1491-9.

5. Harwood JL. Fatty acid metabolism. Annu Rev Plant Physiol Plant Mol Biol. 1988:39:101-38.

6. Patel VK, Shanklin J, Furtek DB. Changes in fatty-acid composition and stearoyl-acyl carrier protein desaturase expression in developing Theobroma cacao L. embryos. Planta. 1994;193(1):83-8.

7. Luo T, Peng SM, Deng WY, Ma DW, Xu Y, Xiao M, Chen F. Characterization of a new stearoyl-acyl carrier protein desaturase gene from Jatropha curcas. Biotechnol Lett. 2006:28(9):657-62.

8. Shanklin J, Cahoon EB. Desaturation and related modifications of fatty acids. Annu Rev Physiol Plant Mol Biol. 1998;49:611-41.

9. Lindqvist $Y$, Huang WJ, Schneider G, Shanklin J. Crystal structure of $\Delta^{9}$ stearoyl-acyl carrier protein desaturase from castor seed and its relationship to other di-iron proteins. EMBO J. 1996;15(16):4081-92.

10. Luo T, Deng WY, Zeng J, Zhang FL. Cloning and characterization of a stearoyl-acyl carrier protein desaturase gene from Cinnamomum longepaniculatum. Plant Mol Biol Rep. 2009;27(1):13-9.

11. Byfield $G, X u e ~ H$, Upchurch $R$. Two genes from soybean encoding soluble delta 9 stearoyl-ACP desaturases. Crop Sci. 2006;46(2):840-6.

12. Whittle $\mathrm{E}$, Cahoon EB, Subrahmanyam S, Shanklin J. A multifunctional acylacyl carrier protein desaturase from Hedera helis L. (English ivy) can synthesize 16- and 18-carbon monoene and diene products. J Biol Chem. 2005;280(31):28169-76.

13. Knutzon DS, Thompson GA, Radke SE, Johnson WB, Knauf VC, Kridl JC Modification of Brassica seed oil by antisense expression of a stearoyl-acyl carrier protein desaturase gene. Proc Natl Acad Sci. 1992;89(7):2624-8.

14. Zaborowska Z, Starzycki M, Femiak I, Swiderski M, Legocki A. Yellow lupine gene encoding stearoyl-ACP desaturase-organization, expression and potential application. Acta Biochim Pol. 2002;49(1):29-42.

15. Goldman I, Rocheford TR, Dudley JW. Molecular markers associated with maize kernel oil concentration in an Illinois high protein $\times$ Illinois low protein cross. Crop Sci. 1994;34(4):908-15.

16. Song XF, Song TM, Dai JR, Rocheford TR, Li JS. QTL mapping of kernel oil concentration with high oil maize by SSR markers. Maydica. 2004:49:41-8.

17. Zhang J, Lu XQ, Song XF, Yan JB, Song TM, Dai JR, Rocheford T, Li JS. Mapping quantitative trait loci for oil, starch, and protein concentrations in grain with high-oil maize by SSR markers. Euphytica. 2008;162(3):335-44.

18. Liu ZJ, Yang XH, Fu Y. SAD, a stearoyl-acyl carrier protein desaturase highly expressed in high-oil maize inbred lines. Russ J Plant Physiol. 2009:56(5):709-15.

19. Hu JY. Identification and expression analysis of $\Delta^{9}$ Searoyl-ACP desaturase genes in maize. Beijing: China Agricultural University; 2008.

20. Yang XH. Dissection on genetic basis of oil in maize grain using linkage and association analysis. Beijing: China Agricultural University; 2008.

21. Rossal M, Smith M, Kunst L. Expression of the FAE1 gene and FAEI promoter activity in developing seeds of Arabidopsis thaliana. Plant Mol Biol. 2001;46(6):717-25.

22. Du HW, Yang XH, Yan JB, Li JS. Fatty acid elongase1 (FAE1) promoter as a candidate for genetic engineering of fatty acids to improve seed oil composition. Afr J Biotechnol. 2011;10(84):19615-22.

23. Kachroo A, Shanklin J, Whittle E, Lapchyk L, Hildebrand D, Kachroo P. The Arabidopsis stearoyl-acyl carrier protein-desaturase family and the contribution of leaf isoforms to oleic acid synthesis. Plant Mol Biol. 2007;63:257-71. 
24. Shilman F, Brand Y, Brand A, Hedvat I, Hovav R. Identification and molecular characterization of homeologous $\Delta 9$-stearoyl acyl carrier protein desaturase3 genes from the allotetraploid peanut (Arachis hypogaea). Plant Mol Biol Rep. 2011;29(1):232-41.

25. Liu Q, Singh S, Green A. Hige-oleic and high-stearic cottonseed oils: nutritionally improved cooking oils developed using gene silencing. J AM Coll Nutr. 2002;21:205-11.

26. Stoutjesdijk PA, Hurlestone C, Singh SP, Green AD. High-oleic acid Australian Brassica napus and $B$. juncea vareties produced by co-suppression of endogenous $\Delta 12$-desaturases. Biochem Soc Trans. 2000;28:938-40.

27. Liu Q, Singh SP, Green AG. High-stearic and high-oleic cottonseed oils produced by hairpin RNA-mediated post-transcriptional gene silencing. Plant Physiol. 2002;129(4):1732-43.

28. Stoutjesdijk PA, Singh SP, Liu Q, Hurlstone C, Waterhous P, Green A. HpRNA-mediated targeting of the Arabidopsis FAD2 gene gives highly efficient and stable silencing. Plant Physiol. 2002;129(4):1723-31.

29. An G, Ebert PR, Mitra A, Ha SB. Binary vectors. In: Gelvin SB, Schilperoot RA, editors. Plant Mol. Biology Manual. P. A3rd ed. The Netherlands: Martinus Nijhoff Dordrecht; 1988. p. 1-19.

30. Clough S, Bent AF. Floral dip: a simplified method for Agrobacteriummediated transformation of Arabidopsis thaliana. Plant J. 1998;16(6):735-43.

31. Frame BR, Zhang HY, Cocciolone SM, Sidorenko LV, Dietrich CR, Pegg SE, Zhen SF, Schnable PS, Wang K. Production of transgenic maize from bombarded type II callus: effect of gold particle size and callus morphology on transformation efficiency. In Vitro Cell Dev-PI. 2000;36(1):21-9.

32. Sukhija PS, Palmquist DL. Rapid method for determination of total fatty acid content and composition of feedstuffs and feces. J Agric Food Chem. 1988;36(6):1202-6.

33. Chenna R, Sugawara H, Koike T, Lopez R, Gibson TJ, Higgins DG, Thompson JD. Multiple sequence alignment with the Clustal series of programs. Nucleic Acids Res. 2003;31:3497-500.

34. Tamura K, Dudley J, Nei M, Kumar S. MEGA4: molecular evolutionary genetics analysis (MEGA) software version 4.0. Mol Biol Evol. 2007;24:1596-9.

\section{Submit your next manuscript to BioMed Central and we will help you at every step:}

- We accept pre-submission inquiries

- Our selector tool helps you to find the most relevant journal

- We provide round the clock customer support

- Convenient online submission

- Thorough peer review

- Inclusion in PubMed and all major indexing services

- Maximum visibility for your research

Submit your manuscript at www.biomedcentral.com/submit

C Biomed Central 